\title{
Article \\ DNA Methylation of Pig FUT3 Promoter Alters mRNA Expression to Regulate E. coli F18 Susceptibility
}

\author{
Zhengchang $\mathrm{Wu}^{1,2}$, Dongfeng Shi ${ }^{1}$, Jian Jin ${ }^{2}$, Hairui Fan ${ }^{1} \oplus$, Wenbin Bao ${ }^{1,3}$ and Shenglong $\mathrm{Wu}^{1,3, *}$ \\ 1 Key Laboratory for Animal Genetics, Breeding, Reproduction and Molecular Design of Jiangsu Province, \\ College of Animal Science and Technology, Yangzhou University, Yangzhou 225009, China; \\ zcwu@yzu.edu.cn (Z.W.); shijingyzu@163.com (D.S.); fanhairui0328@163.com (H.F.); \\ wbbao@yzu.edu.cn (W.B.) \\ 2 College of Veterinary Medicine, Yangzhou University, Yangzhou 225009, China; jianj1127@163.com \\ 3 Joint International Research Laboratory of Agriculture \& Agri-Product Safety, Ministry of Education of China, \\ Yangzhou University, Yangzhou 225009, China \\ * Correspondence: slwu@yzu.edu.cn; Tel.: +86-514-8797-9316
}

Citation: Wu, Z.; Shi, D.; Jin, J.; Fan, H.; Bao, W.; Wu, S. DNA Methylation of Pig FUT3 Promoter Alters mRNA Expression to Regulate E. coli F18 Susceptibility. Genes 2021, 12, 1586. https://doi.org/10.3390/ genes12101586

Academic Editor: Clifford J. Steer

Received: 27 August 2021

Accepted: 2 October 2021

Published: 9 October 2021

Publisher's Note: MDPI stays neutral with regard to jurisdictional claims in published maps and institutional affiliations.

Copyright: (c) 2021 by the authors. Licensee MDPI, Basel, Switzerland. This article is an open access article distributed under the terms and conditions of the Creative Commons Attribution (CC BY) license (https:// creativecommons.org/licenses/by/ $4.0 /)$.

\begin{abstract}
Post-weaning diarrhea (PWD) is frequently associated with E. coli F18 infections in piglets. However, the underlying molecular mechanism concerning the resistance of E. coli F18 in local weaned piglets in China is not clearly understood. In the present study, by a comparative analysis of the transcriptome, a-1,3-fucosyltransferase (FUT3) was evaluated as a key candidate correlated with resistance to E. coli F18 in Sutai and Meishan piglets. Functional analysis demonstrated that FUT3 acts as a key positive regulator of E. coli F18 susceptibility in newly food accustomed piglets. However, the core promoter of FUT3 was present at -500-(-206) bp (chr.2: g.73171117-g.73171616), comprising of 9 methylated $\mathrm{CpG}$ sites. Among these, the methylation levels of the two CpG sites (mC-3, mC-5) located in HIF1A and Sp1 transcription factor (TF) considerably associated with mRNA expression of FUT3 $(p<0.05)$. Our findings indicated that the methylation of $\mathrm{mC}-3$ and $\mathrm{mC}-5$ sites has certain inhibitory effect on FUT3 expression and promotes the resistance of E. coli F18 in piglets. The underlined study may explore FUT3 as a new candidate target in E. coli F18 infection in Chinese local weaned piglets.
\end{abstract}

Keywords: pig; FUT3; expression; DNA methylation; E. coli F18

\section{Introduction}

DNA methylation widely existing in mammals can regulate gene expression and maintain genetic stability with transcription and cell division. Under the catalysis of DNA methyltransferase (DNMTs), S-adenosyl-L-methionine (SAM) as methyl group is added to DNA segments and is converted to 5-methylcytosine (5-mC), $\mathrm{N}^{6}$-methyladenine $\left(\mathrm{m}^{6} \mathrm{~A}\right)$, and 7-Methylguanine (7-mG). Among these, 5-mC is the most important methylation modification in mammals. DNA methylation is the main epigenetic mechanism in promoter regions of the eukaryotic genomes that regulate the expression of a gene by enhancing or attenuating the interaction of TF with sequences of DNA in the promoter region [1]. It has been reported that DNA methylation plays a crucial role in maintaining cell function, regulating individual development and disease [2-4]. DNA methylation is considered to be a significant research hotspot in the current field of pig genetics and breeding, relevant studies mainly focus on tissue-specific expression [5,6], cell apoptosis [7], variety differences [8], growth and development [9-11], immune response [12,13]. However, DNA methylation has been rarely reported in expression regulation of pig resistance to pathogenic microorganism infection.

Piglets' bacterial diarrhea is one of the most common intestinal inflammatory diseases that leads to severe financial damages to pig farming on large scale. Enterotoxigenic Escherichia coli F18 ( . coli F18) is the main pathogenic microbe that results in post-weaning 
diarrhea [14]. However, it is evident from the study that receptors for E. coli F18 in the small intestine of a piglet at the brush border of the epithelial cell, are potentially associated with the pathogenicity of E. coli F18 in terms of their expression levels. In addition, the mechanism being involved in E. coli F18 resistance in Chinese local weaned piglets still remains undiscovered. In this study, based on previously obtained Sutai (a new crossbred between Meishan and the Duroc strains) and Meishan piglets, i.e., resistant and sensitive to E. coli $\mathrm{F} 18[15,16]$, we conducted a comparative mRNA sequencing of duodenum tissues and identified a functioning gene, designated a-1,3-fucosyltransferase (FUT3), that may have critically contributed in anti-E. coli F18 infection. FUT3, belonging to the fucosyltransferase family, regulates the formation of $\mathrm{ABH}$ and Lewis antigens and resistance to pathogen infection $[17,18]$. To further investigate the mechanism of FUT3 expression in regulating E. coli F18 resistance, this study systematically verified the linkage between the expression of FUT3 and infection of E. coli F18 at the tissue and cellular levels. Then, the FUT3 core promoter was determined by bioinformatics analysis and dual-luciferase assay, meanwhile, bisulfite amplicon sequencing (BSAS) was used to detect the level of methylation in FUT3 core promoter in intestinal tissues of piglets, i.e., sensitive and resistant to E. coli F18. Then we determined the impact of the methylation sites on the expression of FUT3 mRNA and evaluated considerable transcription factor in the core promoter region. Hence, this study conformed a candidate target for combating vulnerability to E. coli F18 in weaned piglets and further revealed the effect of DNA methylation on gene expression. Our findings aim to provide insights into the molecular mechanism of piglets' anti-E. coli F18 infection and providing the basis for solving the key scientific problem of E. coli-resistant disease breeding in Chinese local pigs.

\section{Results}

2.1. FUT3 Was Evaluated as a New Target to Combat E. coli F18 Infection on the Basis of Comparative Transcriptome Analysis

To determine the molecular mechanism of regulating E. coli F18 resistance in local pig breeds in Chinese indigenous pigs, we conducted a comparative RNA-seq analysis of duodenal tissues from Sutai and Meishan piglets exhibiting sensitivity or resistance to E. coli F18. In Sutai piglets, 238 differentially expressed genes (DEGs) were screened out between piglets that were sensitive or resistant to E. coli F18 ( $p$-value $<0.05$ and I fold change) $\mid<2$ (Figure 1a). Likewise, 310 DEGs were detected in Meishan piglets (Figure 1b). Interestingly, we identified 46 common DEGs between two pig breeds (Figure 1c,d). Among these DEGs, $\alpha$-1,3-fucosyltransferase (FUT3) is involved in glycosphingolipid biosynthesis (KO pathway: ko00601) correlated with the generation of E. coli F18 receptor [19-21], which is probably considered as a novel target to combat $E$. coli F18 infection in piglets.

\subsection{Knockdown of Pig FUT3 Enhances E. coli F18 Resistance}

To analyze the correlation between the expression of FUT3 and E. coli F18 susceptibility in pigs, we analyzed the differential mRNA and protein expression of FUT3 between piglets that were sensitive and resistant to E. coli F18. qPCR evaluation revealed that the FUT3 gene's expression level was considerably elevated in the duodenum and jejunum of piglets that were sensitive to E. coli F18 relative to those that showed resistance $(p<0.01)$, as depicted in Figure 2a. The results were further confirmed by the immunoblotting which revealed that the protein expression of FUT3 was significantly upregulated in a sensitive group, as depicted in Figure 2b.

To further understand how the expression of FUT3 regulated E. coli F18 invasion, the lentivirus-activated RNAi was used to facilitate the attenuation of FUT3 in the intestinal porcine epithelial cells (IPEC-J2). The expression of a GFP was determined in more than $90 \%$ of cells treated with shRNA vector. As indicated in Figure 3a, The knockdown potential of FUT3 in shFUT3-n $(n=1,2,3,4)$ treated IPEC-J2 cells were $39.9 \%, 71.6 \%, 23.1 \%$, and $86.9 \%$, accordingly, relative to non-treated cells (control) and shFUT3-4 treated IPEC-J2 cells were considered for the successive evaluation. The level of Protein expression showed 
consistency with transcription levels (Figure 3b). Therefore, we successfully established the IPEC-J2 cell line with the silencing of FUT3. Besides, we further investigated the effects of FUT3 expression on the interaction of E. coli F18 to IPEC-J2 cells. After FUT3 knockdown, bacteria enumeration (Figure 3c) and relative quantification (Figure 3d) revealed that the adhesion levels of E. coli F18ab/ac-expressing fimbriae to IPEC-J2 cells in the shFUT3 group were considerably reduced than that in control groups $(p<0.01)$. Immunofluorescence assay (Figure 3e), scanning electron microscopy (Figure 3f), and gram staining (Figure 3g) also showed that the distribution of E. coli F18 in the shFUT3 group was markedly higher than that in the control group. These results suggested that FUT3 contributed to regulating E. coli F18 infection and lowered expression level enhances E. coli F18 resistance.

a

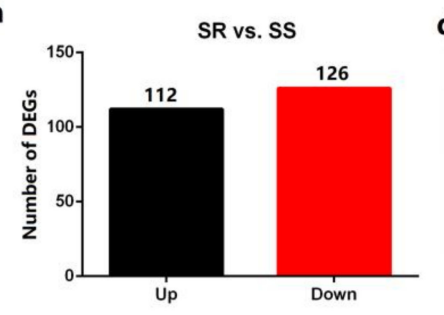

b

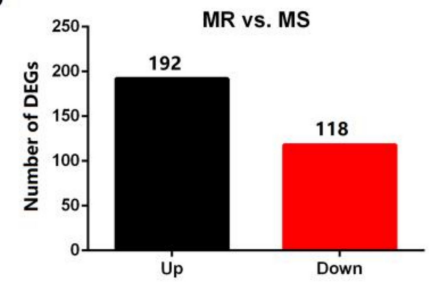

C

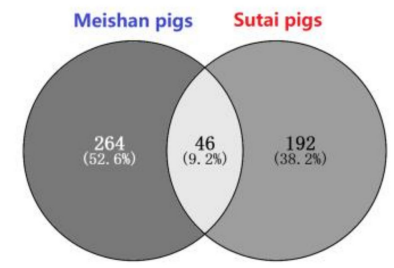

d

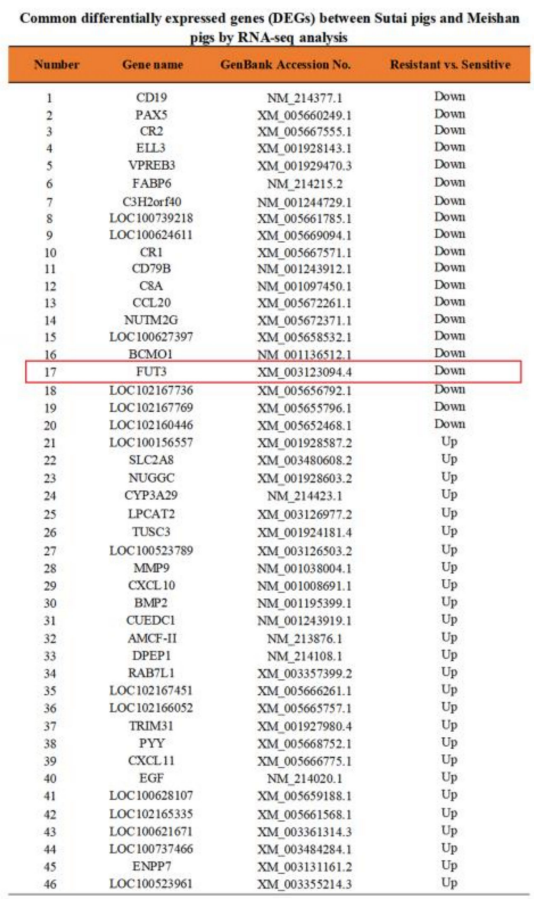

Figure 1. Pig FUT3 is found as a host gene involved in the infection of E. coli F18. (a) DGEs between Sutai F18-resistant (SR) and -sensitive (SS) piglets. (b) DGEs between Meishan F18-resistant (MR) and -sensitive (MS) piglets. (c) Venny screening of common DGEs between Sutai and Meishan piglets. (d) Gene list of common DGEs has been associated with infection caused via E. coli (F18).
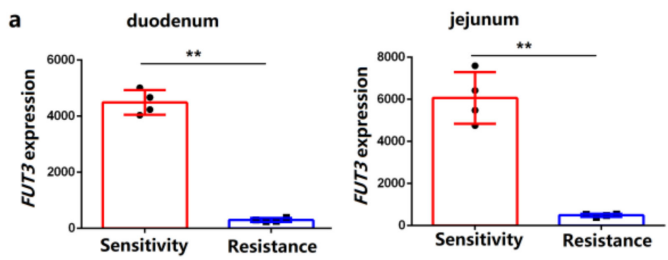

b

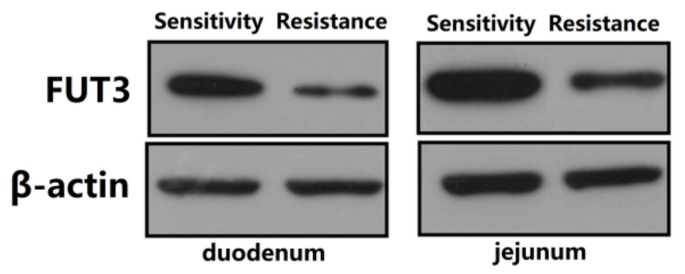

Figure 2. Differential expression analysis of pig FUT3 gene in intestinal tissues between Sutai E. coli F18-resistant and -sensitive piglets. (a) qRT-PCR detection, $n=3$ biological replicates, ${ }^{* *} p<0.01$. (b) Immunoblot analysis, $\beta$-actin, internal reference. 
a

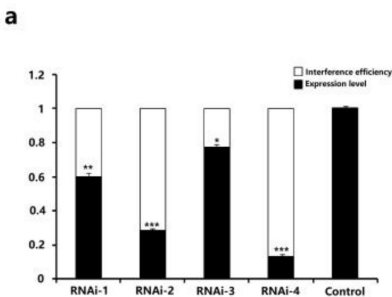

b

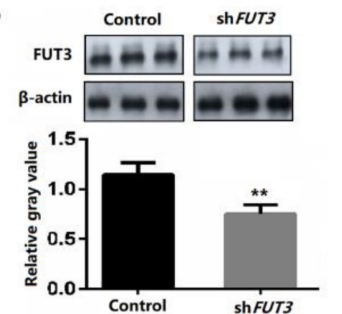

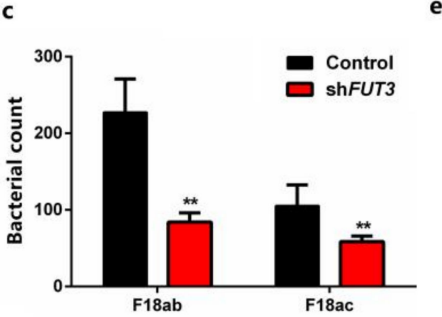

d

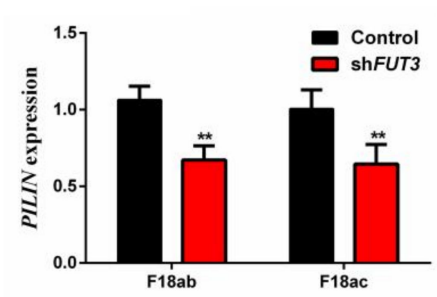

e

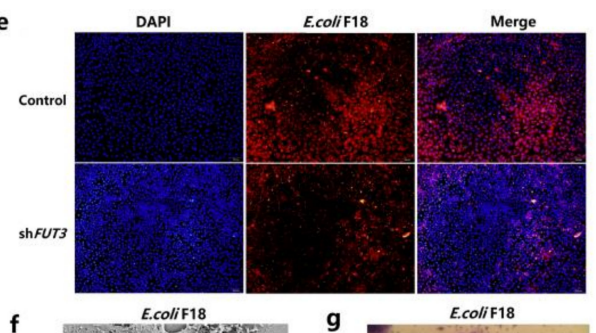

f E.colifis

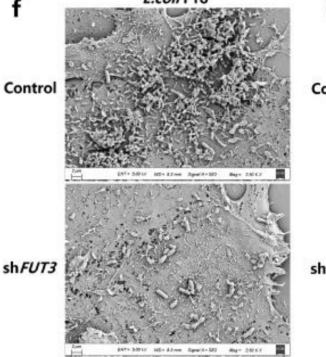

g

E.colif18

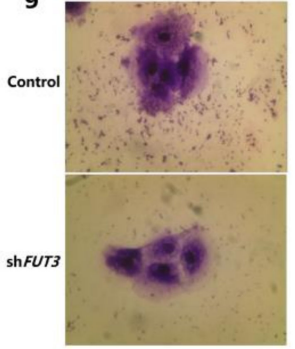

Figure 3. Knockdown of FUT3 enhances E. coli F18 resistance. (a) Interference efficiency evaluation of FUT3 in RNAi-n ( $n=1$, 2, 3, 4) by qRT-PCR. (b) Interference efficiency of FUT3 for RNAi-4 was assessed by immunoblot validation. (c,d) Adhesion of the F18 fimbria to IPEC-J2 cells were evaluated via bacteria enumeration and relative quantification detection, results are represented as mean $\pm \mathrm{SEM}, n=3$ biological replicates, ${ }^{* *} p<0.01$. (e) Immunofluorescence assay. Blue fluorescence indicates nuclear staining via DAPI, red fluorescence indicates E. coli antibody. (f) Scanning electron microscopy (SEM) assay, cells were found under a scanning electron microscope $(2500 \times)$. (g) Gram staining assay. An optical microscope $(400 \times)$ was used to observe cells.

\subsection{Impact of Pig FUT3 Promoter Methylation on Gene Expression}

To further investigate the molecular mechanisms of FUT3 expression, we focused on the DNA methylation modification analysis of pig FUT3 promoter. Firstly, we carried out the determination of the FUT3 core promoter region. According to the IGV analysis of transcriptome sequencing, we achieved a 2000 bp sequence upstream of the FUT3 gene. The prediction of three promoter regions of pig FUT3 was carried out which were present at $-400-(-350) b p,-1297-(-1247) b p,-1848-(-1798) b p$ upstream of the transcription start site. Then, the 2000-bp upstream sequence was divided into four fragments, namely, -200-0 bp (control), -500-0 bp (P1), -1500-0 bp (P2), and -2000-0 bp (P3) (Figure 4a). Agarose gel electrophoresis was employed for evaluating the PCR products, followed by sequencing. PCR amplification products were evaluated via agarose gel electrophoresis $(1 \%)$, as depicted in Figure $4 \mathrm{~b}$. As shown in Figure 4c, luciferase assay revealed that the luciferase intensity of the pRL-P1 was considerably elevated (unpaired $t$-test, $p<0.01$ ) than that of the other transfected groups. We identified that the core promoter region of FUT3 was found at -500-(-200) bp (chr. 2: g.73171117-g.73171616). Based on sequence, we designed the methylation amplification region (Figure $4 \mathrm{~d}$ ) using the MethPrimer software.

Furthermore, we conducted the methylation analysis of the FUT3 promoter in intestinal tissues of individuals that were resistant and sensitive to $E$. coli F18 via bisulfite amplicon sequencing (BSAS). As shown in Figure 5a, we detected nine CpG sites methylated in pig FUT3 core promoter. On the whole, the average methylation levels in the duodenum $(78.25 \%)$ and jejunum (86.63\%) tissues from E. coli F18-resistant individuals were considerably elevated than that in the duodenum $(72.20 \%)$ and jejunum $(75.15 \%)$ tissues from E. coli F18-sensitive individuals $(p<0.05)$. From single CpG sites, the methylation levels of $\mathrm{mC}-5$ and $\mathrm{mC}-6$ sites in intestinal tissues from individuals that were resistant to $E$. coli F18 have considerably elevated than that from E. coli F18-sensitive individuals $(p<0.05$ or $p<0.01$, Figure $5 \mathrm{~b}, \mathrm{c})$. Interestingly, Pearson correlation analysis revealed that the methylation level of the $\mathrm{CpG}$ sites remained in a negative correlation with the mRNA expression of FUT3 (Figure $5 \mathrm{~d}, \mathrm{R}=-0.643, p<0.05$ ), with considerable correlation coefficients being attained only for the $\mathrm{mC}-3(\mathrm{R}=-0.53, p<0.05), \mathrm{mC}-5(\mathrm{R}=-0.818$, 
$p<0.01)$ and $\mathrm{mC}-6$ sites $(\mathrm{R}=-0.83, p<0.01)$, indicating the methylation of $\mathrm{CpG}-(3,5,6)$ sites probably inhibit FUT3 mRNA expression.

a
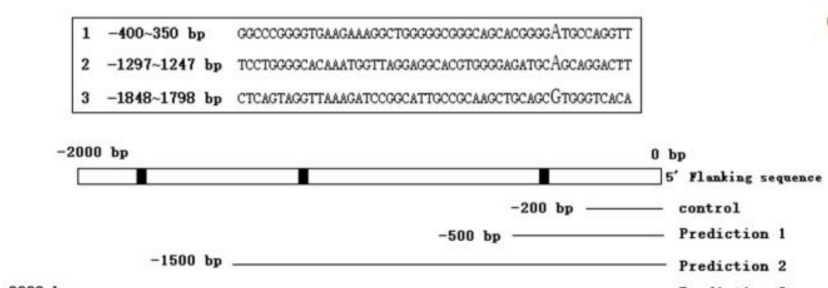

b

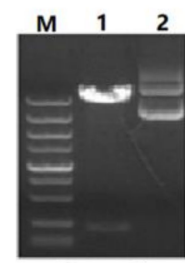

Control

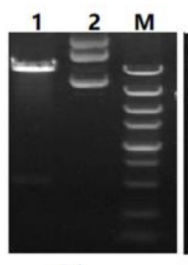

P1

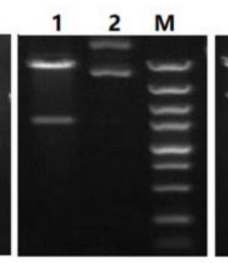

P2

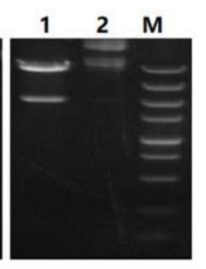

P3
C

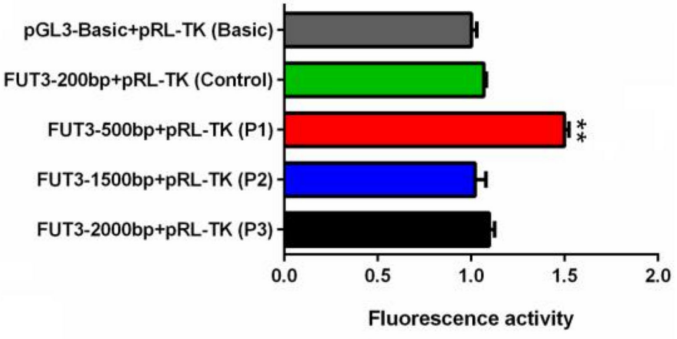

d GCCTGAAATtTAAGCCCCATGAACOCGGCTGGGCTGGGGTGCCAGGGG

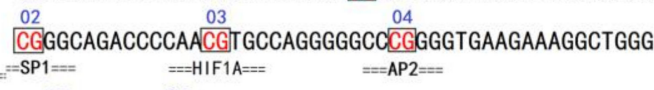
GG: 05 CGGGAGCACGGGGATGCCAGGTTTCTGTCACCTTGTGTTCCCCCC $==\mathrm{SP} 1====\mathrm{USF}=$

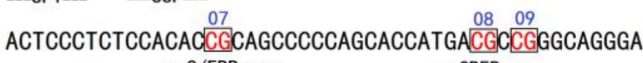
$==\mathrm{C} / \mathrm{EBPa}==\mathrm{CREB}=$

GAGGCTGCATTCTTTAC

Figure 4. Identification of pig FUT3 gene core promoter region. (a) prediction of FUT3 core promoter region and truncation of detection fragment. (b) Agarose gel electrophoresis of PCR products that were digested by restriction enzymes. Lane 1: Plasmid digested by KpnI and HindIII, Lane 2: Plasmid DNA, Lane M: DL5000 Marker. (c) Luciferase assay of different vectors. Basic: negative control. Results are indicated as mean \pm SEM, $n=3$ biological replicates, ${ }^{* *} p<0.01$. (d) Methylation amplification fragment was designed using the MethPrimer software. Box labeled means the methylated CpG sites, below box means the predicted transcription factors.

a

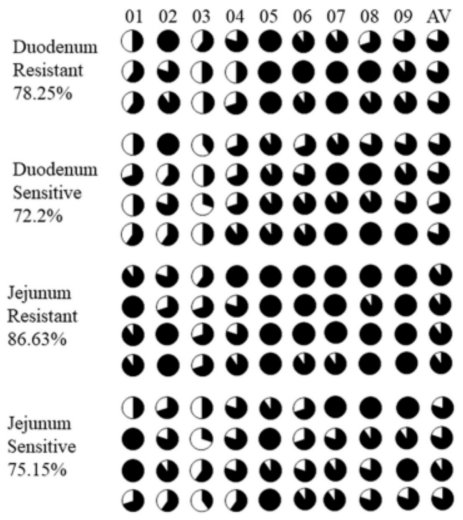

b

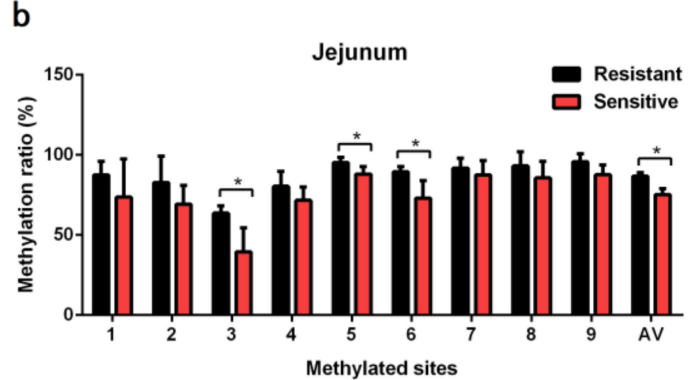

$0 \%$

(1) $\sim 10 \%$

(-) $\sim 20 \%$

(-) $\sim 30 \%$

( $\sim 40 \%$

(1) $\sim 50 \%$

(1) $\sim 60 \%$

- $\sim 70 \%$

- $~ 80 \%$

(1) $\sim 90 \%$

$\sim 100 \%$

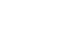

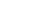

Resistant
C

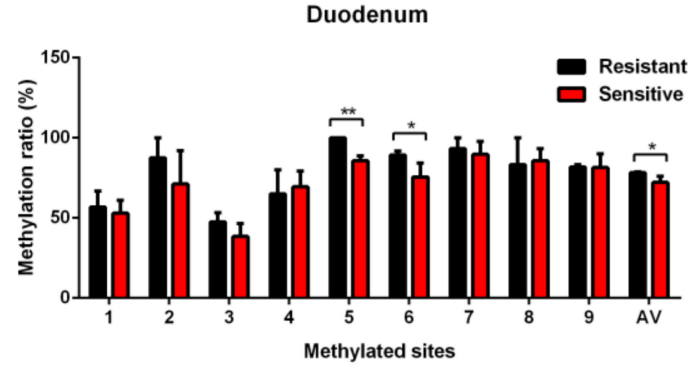

d

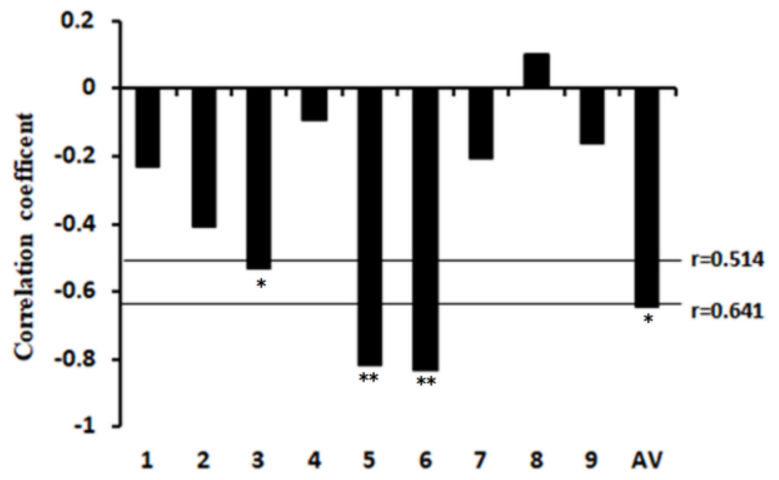

Figure 5. Effect of pig FUT3 promoter methylation level on gene expression. (a) The methylation level of the identified CpG sites. 1 9 mean the different $\mathrm{CpG}$ sites, accordingly. CpG sites are observed with pie charts in which the black region shows the level of methylation. AV: the average degree of methylation. (b) Differential methylation analysis in jejunum tissues from E. coli F18-sensitive and -resistant individuals. (c) Differential methylation analysis in duodenum tissues from E. coli F18-sensitive and -resistant individuals. Results are indicated as mean $\pm \mathrm{SEM},{ }^{*} p<0.05,{ }^{* *} p<0.01$. (d) Correlation analysis between the FUT3 methylation and mRNA expression level. 


\subsection{Evaluation of Key Transcription Factors in Pig FUT3 Core Promoter}

To further identify the important transcription factors in regulating FUT3 expression, we presented the potential transcription factor binding sites (TFBS) found within CpG sites in the core promoter region of pig FUT3 genes, such as SP1, HIF1A, AP2, USF, $\mathrm{C} / \mathrm{EBP} \alpha$, and CREB (Figure $4 \mathrm{~d}$ ). In the above study, we found a significant correlation between the methylation level of CpG-(3, 5, 6) sites and FUT3 mRNA expression (Figure 5d). Furthermore, mC-3, mC-5, and mC-6 were located in the HIF1A (hypoxia-inducible factor $1-\alpha$ ), SP1 (specificity protein 1), and USF (upstream stimulatory factor) transcription factor binding site, which reveals their significant contribution in facilitating expression level of FUT3 by influencing the interaction of TFs with a sequence of the promoter. Herein, we performed dual luciferase activity assay to investigate the effects of HIF1A, SP1, and USF on transcriptional activity in the FUT3 promoter. As shown in Figure 6, HIF1A and SP1 could promote FUT3 transcriptional activity, while USF led to the inhibition of transcriptional activity.

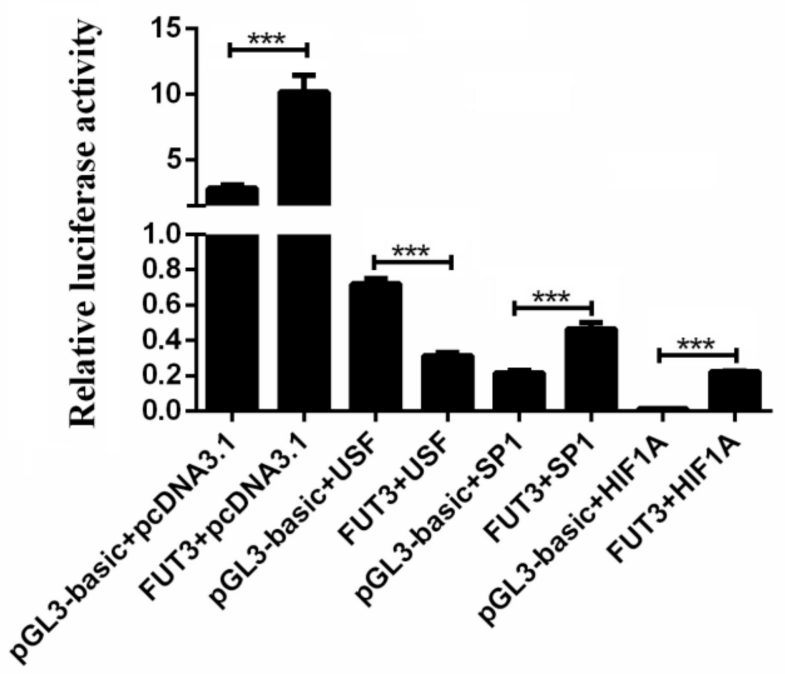

Figure 6. Effects of HIF1A, SP1, and USF on FUT3 transcriptional activity by dual-luciferase activity assay. The obtained results are indicated as mean \pm SEM, ${ }^{* * *} p<0.001$.

\section{Discussion}

To date, no study has assessed the E. coli F18 resistance in Chinese local weaned piglets and their molecular mechanism is still remaining elusive. In previous studies, the TLR genes, i.e., TLR5 and CD14 play important roles in the pig intestine inflammatory response against invading E. coli F18 pathogens [22,23]. In addition to intestinal immunity, resistance to E. coli F18 is most commonly correlated with the expression of the level of its receptor in the intestinal epithelial cells in piglets [24]. According to recent studies, F18-fimbriated $E$. coli interacts with the small intestine of piglets by latching onto glycosphingolipids with blood group ABH determinants on the type- 1 core $[19,20]$. ABH and Lewis antigens (which are the types of Histo-blood group antigens, HBGAs) are formed from precursor antigens, followed by modification by various glycosyltransferases, including a-1,2-fucosyltransferase (FUT2), FUT3 [25]. Herein, we have been carried out a comparative transcriptome analysis of tissue samples taken from the duodenum, followed by identification of a-1,3-fucosyltransferase (FUT3) as a candidate target to battle the susceptibility to E. coli F18 in Chinese local piglets. FUT3 belongs to the family of fucosyltransferase genes and was found to be associated with the mediators of gastrointestinal disease, rotavirus, and bacterial infection $[17,26]$. To investigate the effect of FUT3 expression on E. coli F18 resistance in piglets, qRT-PCR and immunoblotting were carried out to validate the differential expression of FUT3 in intestinal tissues of piglets that were resistant and sensitive to $E$. coli F18. The obtained results indicated a considerably downregulated expression in piglets that were sensitive to E. coli F18. Moreover, we performed an in-vitro functional 
analysis of the E. coli F18 adhesion via RNA interference, followed by demonstrating that FUT3 knockdown markedly enhanced the E. coli F18 adhesion level to intestinal porcine epithelial cells (IPEC-J2). Therefore, it can be speculated that FUT3 expression was involved in the formation of the E. coli F18 receptor and functioned as a positive regulator of enhancing the susceptibility to E. coli F18 in piglets.

To further extensively evaluate the mechanisms that regulate the FUT3 expression alterations in E. coli F18 infected pigs, the methylation of the FUT3 core promoter region in the jejunum and duodenum tissues of piglets (sensitive and resistant to E. coli F18) were evaluated through methylation sequencing. Promoters are often located at 5 'flanking regions, DNA methylation in the regions is considered to be the most obvious epigenetic gene expression regulation. In common, the variations in DNA methylation take place in the CpG [6,11,22], and non-CpG islands [5]. Herein, we examined the 2000 bp upstream of the pig FUT3 gene and observed that there were no CpG islands in this region. In this view, we evaluated the luciferase assay of the successively 5 -shortened sections of FUT3 promoter for the determination of the sequence in the core promoter region $(-500-(-200) b p)$. Our studies showed the higher methylation levels in intestinal tissues of piglets (resistant to E. coli F18) and were found to be exhibited a significant negative correlation $(\mathrm{R}=-0.643, p<0.05)$ between the average methylation level and the expression of mRNA, indicating that FUT3 methylation may inhibit its expression and enhance the immunity of piglets against E. coli F18 infection. Furthermore, multiple studies have revealed that the occurrence of many $\mathrm{CpG}$ sites in the promoter region of a particular gene, but some specific CpG sites can affect gene expression [27]. Analyses of single methylation sites identified a significantly negative association in mRNA expression levels among the mC-3, mC-5, and mC- 6 sites of FUT3 core promoter $(p<0.05)$. In view of these observations, the underlined sites might significantly regulate the transcription of the gene. DNA methylation modulates transcriptional factor (TF) activity by regulating TF binding sites (TFBS) located in the regions with specific CpG sites, leading to the activation or inhibition of gene transcription [6]. Based on transcription factor prediction, we investigated several important transcription factors in FUT3 promoter CpG sites, which belongs to the methylation-dependent transcription factors and include hypoxia-inducible factor (HIF)-1 $\alpha$ (HIF1A) [28], specificity protein 1 (SP1) [29,30], cAMP response elementbinding proteins (CREB) [31], upstream transcription factor (USF) [32], CCAAT/enhancerbinding protein $\beta(\mathrm{C} / \mathrm{EBP} \beta)$ [6]. Interestingly, these $\mathrm{mC}-3, \mathrm{mC}-5$, and $\mathrm{mC}-6$ sites with inhibiting FUT3 expression were located in HIF1A, SP1, and USF, respectively. The dualLuciferase assay showed, HIF1A and SP1 could promote FUT3 transcriptional activity, while USF caused the inhibition of transcriptional activity. Taken together, we revealed that the elevated level of methylation at $\mathrm{mC}-3$ and $\mathrm{mC}-5$ regions overcome the SP1 and HIF1A binding potencies to the FUT3 promoter to suppress the gene expression. In future studies, there is a need for validation of HIF1A and SP1 binding to the FUT3 promoter via electrophoretic mobility shift assays (EMSA) and chromatin-immunoprecipitation (ChIP), meanwhile, we will explore in-depth the function of a key transcription factor by RNA overexpression and CRISPR/CAS9-knockout.

\section{Conclusions}

In conclusion, the present study identified FUT3 as a key target for combating susceptibility to E. coli F18 in Chinese local pigs based on comparative transcriptome analysis. Functional verification indicated that the low expression of FUT3 was linked with resistance to E. coli F18 in piglets. Besides, we determined the FUT3 core promoter region $(-500-(-200 \mathrm{bp})$ by bioinformatics analysis and luciferase assay. Methylation analysis showed, two key CpG sites (mC-3, mC-5) probably decreased the binding activity of HIF1A and SP1 to the promoter of FUT3 for the inhibition of gene expression. Our obtained results may explore the regulatory mechanism of the pig FUT3 gene that affects resistance to the infection caused by E. coli F18 and involved in its application in molecular breeding against bacterial diarrhea in local piglets of China. 


\section{Materials and Methods}

\subsection{Ethics Statement}

The IACUC of Yangzhou University provided approval for all animals conducted studies, under the permit number: SYXK (Su) IACUC 2012-0029. All piglets related experiments were conducted on the directions of administration and regulatory affairs regarding the approval of Experimental Animals by the State Council of the People's Republic of China.

\subsection{Experimental Sample}

Experimental pigs (Meishan, Sutai) were acquired from Kunshan Conservation Ltd. and Sutai Pig Breeding Center. In the previous study, the model animals, i.e., weaning piglets were used to check their vulnerability to E. coli F18 by challenging with the E. coli through feeding strains, i.e., F18ab and F18ac [16]. Based on functional adhesion and receptor binding assays for the type $\mathrm{V}$ secretion system established by our research team [33], three pairs of piglets (resistant and sensitive to E. coli F18) with similar birth weight, coat color, the shape of the body and weaning weight were chosen for the analysis of RNA-seq. All experimental piglets were euthanized via intravenous injection of pentobarbital sodium and duodenum, liver, heart, spleen, kidney, lung, stomach, kidney, muscle, lymph node, thymus, and jejunum tissues were collected, followed by storing in liquid nitrogen in situ for further use.

\subsection{Transcriptome Sequencing (RNA-seq)}

Total RNA of duodenum tissues from 6 Meishan piglets (Resistant group: MR-1, MR-2, MR-3; Sensitive group: MS-1, MS-2, MS-3) and 6 Sutai piglets (Resistant group: SR-1, SR-2, SR-3; Sensitive group: SS-1, SS-2, SS-3) was isolated by TRIzol ${ }^{\mathrm{TM}}$ reagent (Invitrogen, ThermoFisher Scientific, Carlsbad, CA, USA) on the basis of suggestion provided by the manufacturer. The removal of rRNAs from total RNA was carried out via NEBNext rRNA Depletion Kit (USA), as suggested by the manufacturer. Libraries of RNA were formed via the NEBNext ${ }^{\circledR}$ Ultra ${ }^{\mathrm{TM}}$ II Directional RNA Library Prep Kit (USA), as suggested by the manufacturer. Libraries quality and quantification were carried out via the BioAnalyzer 2100 system (USA). RNA Sequencing was conducted at the Novogene Bioinformatics Institute (Beijing, China) on an Illumina Hiseq 2500 platform and $100 \mathrm{bp}$ paired-end reads were constructed. The obtained raw RNA-seq data (high-throughput) were submitted to NCBI (BioProject ID: PRJNA476718, PRJNA476720, PRJNA476721, PRJNA476722).

Paired-end reads were collected from Illumina HiSeq 2500 sequencer, and their quality was controlled via Q30. Post 3' adaptor-trimming and reads (low quality) removal by cutadapt (version 1.9.3), the clean reads with higher quality were aligned to the reference genome (SusScr11) with the software, i.e., hisat2 (version 2.0.4). Then, the cuffdiff software was employed to obtain the gene level FPKM as the expression profiles of mRNA, and a threshold for considerably variant expression was set as a $p<0.05$ and $\mid \log 2$ (fold change) $\mid>1$.

\subsection{Knockdown Analysis}

Four RNAi sequences targeting FUT3 mRNA (R1, R2, R3, R4) and one off-target control (NC) sequence were designed (Table 1) and individually cloned into LV3-H1/GFP\&Puro vector (GenePharma), followed by co-transfection with packaging plasmids into 293T cells (GenePharma). The collection of the virus was carried out that was used for infection of the target cells (IPEC-J2). Positive cells were chosen by adding puromycin $(10 \mu \mathrm{g} / \mathrm{mL})$. After 2 days of transfection, total RNA of various treatment groups was isolated via Trizol. PCR was conducted to determine the target gene expression in cells. Based on FUT3 expression, the lentivirus with the elevated interference potential was chosen. 
Table 1. Primer sequence of pig FUT3 shRNAs.

\begin{tabular}{|c|c|}
\hline Name. & Sequence of the Oligo $\left(5^{\prime} \rightarrow 3^{\prime}\right)$ \\
\hline $\mathrm{R} 1 \mathrm{~F}$ & GATCCggtctggttcagcatggaatcTTCAAGAGAgattccatgctgaaccagaccTTTTTTG \\
\hline R1R & AATTCAAAAAAggtctggttcagcatggaatcTCTCTTGAAgattccatgctgaaccagaccG \\
\hline $\mathrm{R} 2 \mathrm{~F}$ & GATCCgcagggactctgatatcttcaTTCAAGAGAtgaagatatcagagtccctgcTTTTTTG \\
\hline $\mathrm{R} 2 \mathrm{R}$ & AATTCAAAAAAgcagggactctgatatcttcaTCTCTTGAAtgaagatatcagagtccetgcG \\
\hline $\mathrm{R} 3 \mathrm{~F}$ & GATCCgctcaacatctcggccaagaaTTCAAGAGAttcttggccgagatgttgagcTTTTTTG \\
\hline R3R & AATTCAAAAAAgctcaacatctcggccaagaaTCTCTTGAAttcttggccgagatgttgagcG \\
\hline $\mathrm{R} 4 \mathrm{~F}$ & GATCCgacctccaagtggacgtgtatgTTCAAGAGAcatacacgtccacttggaggtcTTTTTTG \\
\hline $\mathrm{R} 4 \mathrm{R}$ & AATTCAAAAAAgacctccaagtggacgtgtatgTCTCTTGAAcatacacgtccacttggaggtcG \\
\hline $\mathrm{NC}-\mathrm{F}$ & GATCCttctccgaacgtgtcacgtTTCAAGAGAagttagttgggactttgttgcTTTTTTG \\
\hline NC-R & AATTCAAAAAAttctccgaacgtgtcacgtTCTCTTGAAagttagttgggactttgttgcG \\
\hline
\end{tabular}

The italic is the introduced enzyme loci, the lowercase is the interference sequence and complementary sequence, and underline represents the loop sequence.

\subsection{Adhesion Level Detection of E. coli F18 to IPEC-J2 In Vitro}

Intestinal porcine epithelial cells (IPEC-J2, provided by the University of Pennsylvania, USA) were grown in medium, i.e., DMEM/F12 in a 1:1 ratio provided with FBS (10\%). For the IPEC-J2 control (Control), FUT3 knockdown cells (shFUT3), in vitro evaluation of F18ab fimbriae standard strain 107/86 (O139:K12:H1) and F18ac (8199) adhesion was performed as explained earlier [33]. To establish an effective and precise method for the detection of $E$. coli adhesion, a relative quantification method [34], bacteria enumeration, gram staining, scanning electron microscopy (SEM), immunofluorescence assay (IFA) [22] were used for measuring E. coli F18 interacting ability with epithelial cells of the small intestine in pigs in vitro.

\subsection{RT-qPCR Analysis}

Total RNA from IPEC-J2 cells and tissues was isolated with the help of Trizol Reagent. The purity, as well as total RNA concentration, were evaluated via agarose gel electrophoresis in formaldehyde (1\%) and NanoDrop 1000 (USA), then RNA was kept at $-70{ }^{\circ} \mathrm{C}$. Synthesis of cDNA was carried out via $1 \mu \mathrm{L}$ of $5 \times$ qRT SuperMix II along with total RNA of $500 \mathrm{ng}$, and $10 \mu \mathrm{L}$ of RNase free $\mathrm{ddH}_{2} \mathrm{O}$. RT-qPCR instrument ABI7500 was employed for the evaluation of qPCR and the parameters were set as $95{ }^{\circ} \mathrm{C}$ for 30 and $5 \mathrm{~s}$, accordingly, followed by $60^{\circ} \mathrm{C}$ for $34 \mathrm{~s}$, and then 40 cycles were carried out. The primers of amplified fragments of FUT3 and $\beta$-actin were indicated in Table 2.

Table 2. Real-time PCR primers and sequences.

\begin{tabular}{|c|c|c|c|}
\hline Gene Name & $\begin{array}{c}\text { GenBank } \\
\text { Accession Number }\end{array}$ & Primer Sequence & Fragment Size \\
\hline FUT3 & AF130972.1 & $\begin{array}{l}\text { F: 5'-CCCGAAGCCTTCATCCACAT-3' } \\
\text { R: } 5^{\prime} \text {-CATCAAGGCCCAGCTGAAGA-3' }\end{array}$ & $150 \mathrm{bp}$ \\
\hline PILIN & M25302.1 & $\begin{array}{l}\text { F: 5'-AGGCCGAACCAAAGAAGCAT-3' } \\
\text { R: } 5^{\prime} \text {-TCACCATCAGGGTTTCTGAGT-3' }\end{array}$ & $117 \mathrm{bp}$ \\
\hline$\beta$-actin & NC_010445.3 & $\begin{array}{c}\text { F:GTCGTACTCCTGCTTGCTGAT } \\
\text { R:CCTTCTCCTTCCAGATCATCGC }\end{array}$ & $119 \mathrm{bp}$ \\
\hline
\end{tabular}

\subsection{Determination of Pig FUT3 Core Promoter Region}

The 2000-bp upstream sequence of the transcription start site of pig FUT3 gene was obtained by NCBI and UCSC database. The prediction of CpG islands of the pig FUT3 upstream region was performed via the MethPrimer software. Besides, the prediction of the FUT3 core promoter region was carried out via BDGP software. Based on the above bioinformatics analysis, the 2000-bp upstream sequence was designed for the amplification of various promoter fragments truncated at the $3^{\prime}$ end of the sequence. Then, the resulted PCR products were evaluated via agarose gel electrophoresis, then sequencing was carried 
out. Recombinant vectors were labeled as FUT3-200bp (Control), FUT3-500bp (P1), FUT31500bp (P2), FUT3-2000bp (P3), and then co-transfected with pRL-TK vector into cells for dual luciferase assay.

\subsection{Detection of Methylation in Pig FUT3 Core Promoter Region}

Genomic DNA from duodenum and jejunum tissues was transformed via EpiTect Fast DNA Bisulfite kit (QIAGEN, Hilden Germany), and the underlined PCR primers (F:GTTTGAAATTTAAGTTTTATGAATT,R:ATAAAAAATACAACCTCTCCCTACC) were established on the basis of the sequence of the sulfite transformation via the MethPrimer software. Moreover, 25- $\mu \mathrm{L}$ PCR amplification reactions of bisulfite-treated DNA (BSTDNA) consist of $2 \mu \mathrm{L}$ DNA template, $12.5 \mu \mathrm{L}$ ZYMO Taq Premix, $1 \mu \mathrm{L}$ forward primer $(10 \mu \mathrm{M} / \mathrm{L}), 1 \mu \mathrm{L}$ reverse primer $(10 \mu \mathrm{M} / \mathrm{L}), 8.5 \mu \mathrm{L}$ distilled $\mathrm{DI}_{2} \mathrm{H}_{2} \mathrm{O}$. The subsequent reaction conditions were as follows: $95^{\circ} \mathrm{C}$ for $10 \mathrm{~min}$, then 35 cycles of $95^{\circ} \mathrm{C}$ for $30 \mathrm{~s}, 52^{\circ} \mathrm{C}$ for $30 \mathrm{~s}, 72{ }^{\circ} \mathrm{C}$ for $35 \mathrm{~s}$, and $72{ }^{\circ} \mathrm{C}$ for $10 \mathrm{~min}$. The TIANquick Midi purification kit (Tiangen Biotech, Beijing) was utilized for the purification of PCR products (209 bp), followed by cloning into the pMD19-T vector (Takara, Dalian, China). A total of 30 positive clones were randomly chosen for sequencing of all samples (Invitrogen Biotechnology, Shanghai, China) and sequence data for methylation level were evaluated via the quantification tool for methylation analysis (QUMA) software [35].

\subsection{Identification of Key Transcription Factor in Pig FUT3 Core Promoter Region}

Alibaba (http:/ /www.gene-regulation.com/pub/programs/alibaba2/index.html?, accessed on 18 February2021) and JASPAR (http://jaspar.genereg.net/cgi-bin/jaspar_db. $\mathrm{pl}$, accessed on 18 February 2021) were employed to evaluate transcription factor binding sites (TFBS), basing on the sequence of the FUT3 promoter region. Based on the CpG sites of methylation level significantly altering mRNA expression, we further screened out the important transcription factors and conducted validation experiments by dual-luciferase activity assay.

\subsection{Statistical Analyses}

In order to conduct statistical analyses, the SPSS 18.0 software (Chicago, IL, USA) was used. Relative quantitative results were examined via the $2^{-\Delta \Delta \mathrm{Ct}}$ method [36]. Results were represented as mean \pm SEM, followed by comparison via Student's $t$-test. $p<0.05$ or $p<0.01$ indicated statistical significance. Pearson's correlation was performed to evaluate the association between FUT3 promoter methylation and mRNA expression.

Author Contributions: Conceived and designed the experiments: W.B. and S.W. Performed the experiments: Z.W., D.S., and J.J. Analyzed the data: Z.W. Contributed reagents/materials/analysis tools: H.F. Contributed to the writing of the manuscript: Z.W. and D.S. All authors have read and agreed to the published version of the manuscript.

Funding: This study was supported by Jiangsu Agricultural Science and Technology Innovation Fund (CX(20)3011), Natural Science Foundation of Jiangsu province, China (Grant No. BK20180899), Key Research and Development Project (Modern Agriculture) of Jiangsu Province (BE2019341), National Natural Science Funds (31772560), the earmarked fund for Jiangsu Agricultural Industry Technology System and the Priority Academic Program Development of Jiangsu Higher Education Institutions.

Institutional Review Board Statement: The study was conducted according to the guidelines of the Declaration of the State Council of the People's Republic of China, and approved by the Ethics Committee of Yangzhou University (permit number: SYXK (Su) IACUC 2012-0029; Approval Date: 9 December 2012).

Informed Consent Statement: Not applicable.

Data Availability Statement: Transcriptome sequencing data has been submitted to NCBI's SRA repository under BioProject IDs: PRJNA476718, PRJNA476720, PRJNA476721, PRJNA476722. 
Acknowledgments: We thank Novogene Bioinformatics Technology, Beijing, China for Illumina sequencing.

Conflicts of Interest: The authors declare that they have no conflict of interests.

$\begin{array}{ll}\text { Abbreviations } \\ \text { E. coli } & \text { Escherichia coli } \\ \text { FUT3 } & \text { a-1,3-fucosyltransferase } \\ \text { PWD } & \text { Post-weaning diarrhea } \\ \text { TF } & \text { transcription factor } \\ \text { Sp1 } & \text { Specificity protein 1 } \\ \text { HIF1A } & \text { hypoxia-inducible factor 1- } \alpha \\ \text { USF } & \text { upstream stimulatory factor } \\ \text { RNA-seq } & \text { RNA sequencing } \\ \text { DEGs } & \text { differentially expressed genes } \\ \text { SEM } & \text { Scanning electron microscopy } \\ \text { BSAS } & \text { bisulfite amplicon sequencing } \\ \text { IGV } & \text { Integrative Genomics Viewer } \\ \text { TFBS } & \text { transcription factor binding sites }\end{array}$

\section{References}

1. Bender, J. DNA methylation and epigenetics. Annu. Rev. Plant Biol. 2004, 55, 41-68. [CrossRef] [PubMed]

2. Flores, E.M.; Woeller, C.F.; Falsetta, M.L.; Susiarjo, M.; Phipps, R.P. Thy1 (CD90) expression is regulated by DNA methylation during adipogenesis. FASEB J. 2018, 33, 3353-3363. [CrossRef] [PubMed]

3. Zeng, Y.; Chen, T. DNA Methylation Reprogramming during Mammalian Development. Genes 2019, 10, 257. [CrossRef] [PubMed]

4. Greenberg, M.V.C.; Bourc'his, D. The diverse roles of DNA methylation in mammalian development and disease. Nat. Rev. Mol. Cell Biol. 2019, 20, 590-607. [CrossRef]

5. Cao, Y.; Gao, Z.C.; Wu, Z.C.; Wang, H.F.; Bao, W.B. Tissue-specific expression and correlation with promoter DNA methylation of the LBP gene in pigs. J. Integr. Agric. 2020, 19, 1054-1063. [CrossRef]

6. Sun, L.; Wang, J.; Yin, X.M.; Sun, S.Y.; Zi, C.; Zhu, G.Q.; Wu, S.L.; Bao, W.B. Identification of a 5-Methylcytosine Site that may Regulate C/EBP $\beta$ Binding and Determine Tissue-Specific Expression of the BPI Gene in Piglets. Sci. Rep. 2016, 6, 28506. [CrossRef]

7. Xu, Y.; Chen, X.; Yu, L.; Wang, Y.; Wang, H.; Wu, Z.; Wu, S.; Bao, W. SLC4A11 and MFSD3 Gene Expression Changes in Deoxynivalenol Treated IPEC-J2 Cells. Front. Genet. 2021, 12, 697883. [CrossRef]

8. Wang, H.F.; Wu, J.Y.; Wu, S.; Wu, S.L.; Bao, W.B. DNA methylation differences of the BPI promoter among pig breeds and the regulation of gene expression. RSC Adv. 2017, 7, 48025-48030. [CrossRef]

9. Wang, H.F.; Feng, H.Y.; Sun, J.; Zhou, Y.J.; Zhu, G.Q.; Wu, S.L.; Bao, W.B. Age-associated changes in DNA methylation and expression of the TNF $\alpha$ gene in pigs. Gene Genet. Syst. 2018, 93, 191-198. [CrossRef]

10. Dai, C.; Sun, L.; Xia, R.; Sun, S.; Zhu, G.; Wu, S.; Bao, W. Correlation between the methylation of the FUT1 promoter region and FUT1 expression in the duodenum of piglets from newborn to weaning. 3 Biotech 2017, 7, 247. [CrossRef]

11. Wang, J.; Yin, X.M.; Sun, L.; Sun, S.Y.; Zi, C.; Zhu, G.Q.; Wu, S.L.; Bao, W.B. Correlation between BPI Gene Upstream CpG Island Methylation and mRNA Expression in Piglets. Int. J. Mol. Sci. 2014, 15, 10989-10998. [CrossRef]

12. Zheng, Z.X.; Wan, C.; Mo, D.L.; Li, J.P.; Chen, Y.S.; Zhang, Z.W.; Cong, P. Promoter CpG methylation status in porcine Lyn is associated with its expression levels. Gene 2012, 511, 73-78. [CrossRef]

13. Dong, W.H.; Yin, X.M.; Sun, L.; Wang, J.; Sun, S.Y.; Zhu, G.Q.; Wu, S.L.; Bao, W.B. Age-associated methylation change of TAP1 promoter in piglet. Gene 2015, 573, 70-74. [CrossRef]

14. Boldin, B. Persistence and spread of gastro-intestinal infections: The case of enterotoxigenic Escherichia coli in piglets. Bull. Math. Biol. 2008, 70, 2077-2101. [CrossRef]

15. Ye, L.; Su, X.M.; Wu, Z.C.; Zheng, X.R.; Wang, J.; Zi, C.; Wu, S.L.; Bao, W.B. Analysis of differential miRNA expression in the duodenum of Escherichia coli F18-sensitive and -resistant weaned piglets. PLoS ONE 2012, 7, e437412012. [CrossRef]

16. Wu, Z.C.; Dong, W.H.; Liu, Y.; Yang, J.S.; Zhu, G.Q.; Wu, S.L.; Bao, W.B. Attack Experiment and Phenotype Analysis of Meishan piglets by E. coli F18 Strain. Acta Vet. Zootech. Sin. 2014, 45, 1608-1615. (In Chinese)

17. Heggelund, J.E.; Varrot, A.; Imberty, A.; Krengel, U. Histo-blood group antigens as mediators of infections. Curr. Opin. Struct. Biol. 2017, 44, 190-200. [CrossRef]

18. Jiang, X.; Liu, Y.; Tan, M. Histo-blood group antigens as receptors for rotavirus, new understanding on rotavirus epidemiology and vaccine strategy. Emerg. Microbes Infect. 2017, 6, e22. [CrossRef]

19. Coddens, A.; Diswall, M.; Angström, J.; Breimer, M.E.; Goddeeris, B.; Cox, E.; Teneberg, S. Recognition of blood group ABH type 1 determinants by the FedF adhesin of F18-fimbriated Escherichia coli. J. Biol. Chem. 2009, 284, 9713-9726. [CrossRef] 
20. Moonens, K.; Bouckaert, J.; Coddens, A.; Tran, T.; Panjikar, S.; De Kerpel, M.; Cox, E.; Remaut, H.; De Greve, H. Structural insight in histo-blood group binding by the F18 fimbrial adhesin FedF. Mol. Microbiol. 2012, 86, 82-95. [CrossRef]

21. Lonardi, E.; Moonens, K.; Buts, L.; de Boer, A.R.; Olsson, J.D.; Weiss, M.S.; Fabre, E.; Guérardel, Y.; Deelder, A.M.; Oscarson, S.; et al. Structural sampling of glycan interaction profiles reveals mucosal receptors for fimbrial adhesins of enterotoxigenic Escherichia coli. Biology 2013, 2, 894-917. [CrossRef] [PubMed]

22. Dai, C.H.; Yang, L.; Jin, J.; Wang, H.F.; Wu, S.L.; Bao, W.B. Regulation and Molecular Mechanism of TLR5 on Resistance to Escherichia coli F18 in Weaned Piglets. Animals 2019, 9, 735. [CrossRef] [PubMed]

23. Wu, Z.; Liu, Y.; Dong, W.; Zhu, G.Q.; Wu, S.; Bao, W. CD14 in the TLRs signaling pathway is associated with the resistance to $E$. coli F18 in Chinese domestic weaned piglets. Sci. Rep. 2016, 6, 24611. [CrossRef] [PubMed]

24. Shi, Q.S.; Xie, X.M.; Liu, X.C.; Huang, S.Q.; He, C.Q. Experimental results on enterotoxigenic E. coli F18 receptor genotypes. Hereditas 2002, 24, 656-658. (In Chinese)

25. Marionneau, S.; Cailleau-Thomas, A.; Rocher, J.; Le Moullac-Vaidye, B.; Ruvoën, N.; Clément, M.; Le Pendu, J. ABH and Lewis histo-blood group antigens, a model for the meaning of oligosaccharide diversity in the face of a changing world. Biochimie 2001, 83, 565-573. [CrossRef]

26. Hu, D.Y.; Zhang, D.G.; Zheng, S.Z.; Guo, M.D.; Lin, X.X.; Jiang, Y. Association of Ulcerative Colitis with FUT2 and FUT3 Polymorphisms in Patients from Southeast China. PLoS ONE 2016, 11, e0146557. [CrossRef]

27. Xie, X.L.; Yu, Y.; Yuan, Z.F.; Yang, J.; Ma, P.P.; Li, D.C.; Yu, S.K.; An, F.; Feng, X.J.; Zhang, Y. Comparative analysis on content and distribution of $\mathrm{CpG}$ sites in milk production traits and mastitis-related genes in dairy cattle. Hereditas 2012, 34, 437-444. (In Chinese) [CrossRef]

28. Walczak-Drzewiecka, A.; Ratajewski, M.; Pułaski, Ł.; Dastych, J. DNA methylation-dependent suppression of HIF1A in an immature hematopoietic cell line HMC-1. Biochem. Biophys. Res. Commun. 2010, 391, 1028-1032. [CrossRef]

29. Tian, H.P.; Lun, S.M.; Huang, H.J.; He, R.; Kong, P.Z.; Wang, Q.S.; Li, X.Q.; Feng, Y.M. DNA Methylation Affects the SP1-regulated Transcription of FOXF2 in Breast Cancer Cells. J. Biol. Chem. 2015, 290, 19173-19183. [CrossRef]

30. Mancarelli, M.M.; Zazzeroni, F.; Ciccocioppo, L.; Capece, D.; Po, A.; Murgo, S.; Di Camillo, R.; Rinaldi, C.; Ferretti, E.; Gulino, A.; et al. The tumor suppressor gene KCTD11REN is regulated by Sp1 and methylation and its expression is reduced in tumors. Mol. Cancer 2010, 9, 172. [CrossRef]

31. Kim, H.P.; Leonard, W.J. CREB/ATF-dependent T cell receptor-induced FoxP3 gene expression: A role for DNA methylation. J. Exp. Med. 2007, 204, 1543-1551. [CrossRef]

32. Fujii, G.; Nakamura, Y.; Tsukamoto, D.; Ito, M.; Shiba, T.; Takamatsu, N. CpG methylation at the USF-binding site is important for the liver-specific transcription of the chipmunk HP-27 gene. Biochem. J. 2006, 395, 203-209. [CrossRef]

33. Liu, L.; Wang, J.; Zhao, Q.; Zi, C.; Wu, Z.; Su, X.; Huo, Y.; Zhu, G.; Wu, S.; Bao, W. Genetic variation in exon 10 of the BPI gene is associated with Escherichia coli F18 susceptibility in Sutai piglets. Gene 2013, 523, 70-75. [CrossRef]

34. Dai, C.H.; Gan, L.N.; Qin, W.U.; Zi, C.; Zhu, G.Q.; Wu, S.L.; Bao, W.B. Use of fluorescence quantitative polymerase chain reaction (PCR) for the detection of Escherichia coli adhesion to pig intestinal epithelial cells. Pol. J. Vet. Sci. 2016, 19, 303-308. [CrossRef]

35. Kumaki, Y.; Oda, M.; Okano, M. QUMA: Quantification tool for methylation analysis. Nucleic Acids Res. 2008, 36, W170-W175. [CrossRef]

36. Livak, K.J.; Schmittgen, T.D. Analysis of relative gene expression data using real-time quantitative PCR and $2^{-\Delta \Delta C T}$ method. Methods 2001, 25, 402-408. [CrossRef] 